\title{
Association of Plasma Level of TNF-Related Apoptosis-Inducing Ligand with Severity and Outcome of Sepsis
}

\author{
Hongseok Yoo ${ }^{1}$, Jin Young Lee ${ }^{2}$, Junseon Park ${ }^{2}$, Jeong Hoon Yang ${ }^{2,3}$, Gee Young Suh ${ }^{1,2}$ and \\ Kyeongman Jeon $1,2, * \mathbb{D}$ \\ 1 Division of Pulmonary and Critical Care Medicine, Department of Medicine, Samsung Medical Center, \\ Sungkyunkwan University School of Medicine, Seoul 06351, Korea; hs.yoo@skku.edu (H.Y.); \\ suhgy@skku.edu (G.Y.S.) \\ 2 Department of Critical Care Medicine, Samsung Medical Center, Sungkyunkwan University School of \\ Medicine, Seoul 06351, Korea; yenayein@gmail.com (J.Y.L.); study13.park@samsung.com (J.P.); \\ jhysmc@gmail.com (J.H.Y.) \\ 3 Division of Cardiology, Department of Medicine, Samsung Medical Center, Sungkyunkwan University \\ School of Medicine, Seoul 06351, Korea \\ * Correspondence: kjeon@skku.edu
}

Received: 28 April 2020; Accepted: 26 May 2020; Published: 1 June 2020

\begin{abstract}
Recent studies have suggested that TNF-related apoptosis-inducing ligand (TRAIL) is associated with mortality in sepsis, possibly through necroptosis. The objective of this study was to analyze the association between the plasma level of TRAIL and sepsis severity and outcomes. Furthermore, the plasma level of TRAIL was compared to that of receptor-interacting protein kinase-3 (RIPK3), a key executor of necroptosis, to identify any correlation between TRAIL and necroptosis. Plasma levels of TRAIL and RIPK3 from consecutively enrolled critically ill patients were measured by ELISA. Of 190 study patients, 59 (31.1\%) and $84(44.2 \%)$ patients were diagnosed with sepsis and septic shock, respectively. There was a trend of decreased plasma level of TRAIL across the control, sepsis, and septic shock groups. For 143 patients with sepsis, patients with low plasma TRAIL were more likely to have septic shock and higher SAPS3 and SOFA scores. However, no difference in 28-day and 90-day mortalities was observed between the two groups. The plasma level of TRAIL was inversely associated with RIPK3 in patients with sepsis. Plasma levels of TRAIL increased over time on days three and seven, and were inversely associated with sepsis severity and RIPK3 level, but not with mortality.
\end{abstract}

Keywords: biomarker; necroptosis; RIPK3 protein; sepsis; TNF-related apoptosis-inducing ligand

\section{Introduction}

Sepsis is a life-threatening organ dysfunction caused by a dysregulated host response to infection [1] that may culminate in organ failure and death. Despite continuous efforts to understand and improve outcomes of sepsis, it remains a commonly fatal disease [2-4]. In addition, the current definition of sepsis identifies a heterogeneous population of individuals with diverse patterns of immune response, organ dysfunction, and clinical outcomes [1]. Therefore, early diagnosis, precise stratification of severity, and accurate outcome prediction are critical in managing patients with sepsis. Various biomarkers, including C-reactive protein and procalcitonin, have been investigated to date [5,6]; however, no marker has demonstrated sufficient discriminatory power [7].

Tumor necrosis factor (TNF)-related apoptosis-inducing ligand (TRAIL) is a cytokine and member of the TNF superfamily. TRAIL initiates apoptosis of transformed cells or tumor cells by binding 
to death receptors (DR) 4 or 5 [8] and functions as an immune response regulator in sepsis $[8,9]$. More recently, however, it was discovered that TRAIL also functions as a trigger for necroptosis, a specific form of programmed cell necrosis characterized by its caspase-independent activation and release of damage-associated molecular patterns (DAMPs) which convey highly proinflammatory properties $[10,11]$. In a recent study, plasma TRAIL was related to poor outcomes in patients with sepsis [12]. A subsequent multicenter study suggested a relationship between the plasma level of TRAIL with necroptosis in sepsis; however, its association with mortality demonstrated a contradictory result [13]. Therefore, further studies are necessary to clarify the relationship between plasma TRAIL and predicting outcomes of patients with sepsis. In this study, we analyzed the association between the plasma level of TRAIL and severity and outcomes of sepsis. We also compared the plasma level of TRAIL to that of receptor-interacting protein kinase-3 (RIPK3), a well-known necroptosis mediator [14], to identify the correlation between TRAIL and necroptosis.

\section{Materials and Methods}

\subsection{Study Design and Registry}

This was a prospective observational study of the Samsung Medical Center Registry of Critical Illness (SMC RoCI), which is an on-going single-center prospective registry of the Samsung Medical Center (1989-bed, university affiliated, tertiary referral hospital in Seoul, South Korea) initiated in April 2014 for the purpose of establishing a human sample repository and developing new biological markers for critical illness [15]. The study was approved by the institutional review board of Samsung Medical Center. Written informed consent was obtained from patients or their legally authorized representative prior to enrollment.

\subsection{Study Patients}

Critically ill adult ( $\geq 19$ years old) patients admitted to the medical intensive care unit (ICU) of Samsung Medical Center were considered eligible for inclusion in the registry. Exclusion criteria were as follows: (1) cognitive impairment, (2) inability to provide informed consent, (3) ICU admission for a simple procedure or postsurgical care, (4) transfer from other hospitals, (5) end-of-life decision or admission to facilitate comfort care, (6) hemoglobin $<8 \mathrm{~g} / \mathrm{dL}$ upon admission or persistent bleeding, and (7) discharge within $24 \mathrm{~h}$ of admission to ICU. Screening and enrollment were completed within $24 \mathrm{~h}$ of ICU admission. Patients registered between April 2014 and December 2016 were included in the analysis. Some clinical data and RIPK3 levels from patients enrolled until August 2016 were reported in a previous study [15].

\subsection{Data Collection}

A trained study coordinator used hospital records for each patient to prepare a standardized case report form. Clinical data consisting of patient demographics, reason for ICU admission, severity of illness scoring, and laboratory data were obtained at the time of enrollment. Illness severity was assessed by the Acute Physiology and Chronic Health Evaluation II (APACH II) [16], Simplified Acute Physiology Score 3 (SAPS 3) [17], and Sequential Organ Failure Assessment (SOFA) scores [18]. The Revised Trauma Score was used to determine the severity of trauma patients [19]. The primary outcome was 28-day mortality. Secondary outcomes were in-hospital and 90-day mortality.

Sepsis was defined according to the third International Consensus Definitions for Sepsis and Septic Shock (Sepsis-3) [1]. Since enrollment for the registry began in April 2014, patients enrolled before release of the new definition were reclassified. Patients in the registry who did not meet the definition of either sepsis or septic shock were defined as controls. 


\subsection{Measurement of Plasma TRAIL and RIPK3}

Along with clinical data, $19 \mathrm{~mL}$ of whole blood was drawn from each patient within $48 \mathrm{~h}$ of study enrollment. When possible, additional blood samples were collected at day $3(15 \mathrm{~mL})$ and day $7(15 \mathrm{~mL})$. Blood samples were centrifuged within $4 \mathrm{~h}$ of collection. Plasma was separated and stored at $-80^{\circ} \mathrm{C}$ until further analysis. Plasma TRAIL level was measured from stored aliquots using commercially available TRAIL Human ELISA kits according to the manufacturer's recommendations (R \& D systems, Minneapolis, MN, USA). Plasma RIPK3 level was measured using a commercially available ELISA kit as per the manufacturer's recommendations (CUSABIO, Houston, TX, USA) [15].

\subsection{Statistical Analysis}

Data are presented as numbers (percentages) for categorical variables, and as the median and interquartile range (IQR, 25th-75th percentiles) for continuous variables. Categorical variables were compared using the Chi-square test or Fisher's exact test, while continuous variables were compared using the Mann-Whitney $U$ test. Differences in plasma TRAIL level across control, sepsis, and septic shock groups were assessed with the Kruskal-Wallis test. Baseline characteristics, clinical status at ICU admission, illness severity, and mortality were compared between patients with low and high TRAIL level divided by the median level of plasma TRAIL. To evaluate the association between plasma TRAIL level and patient outcomes, a Kaplan-Meier curve was used to determine the 90-day survival curves according to plasma TRAIL. These were then compared using the log-rank test. Linear regression was applied to assess the association between plasma levels of TRAIL and RIPK3. The Friedman test was performed to determine differences among serial levels of TRAIL. The Wilcoxon signed rank test with Bonferroni correction was used to compare plasma TRAIL levels between days 0 and 3 and between days 3 and 7.

All tests were two-sided, and a $p$ value $<0.05$ was considered significant. Data were analyzed using IBM SPSS Statistics 20.0 (IBM, Chicago, IL, USA).

\section{Results}

Over the study period, 1419 patients were admitted to the ICU. After excluding 1224 patients who met the exclusion criteria and one patient who missed screening, 194 patients were enrolled in the registry. Since four patients withdrew their consent, 190 patients were finally included in the analysis (Figure 1).

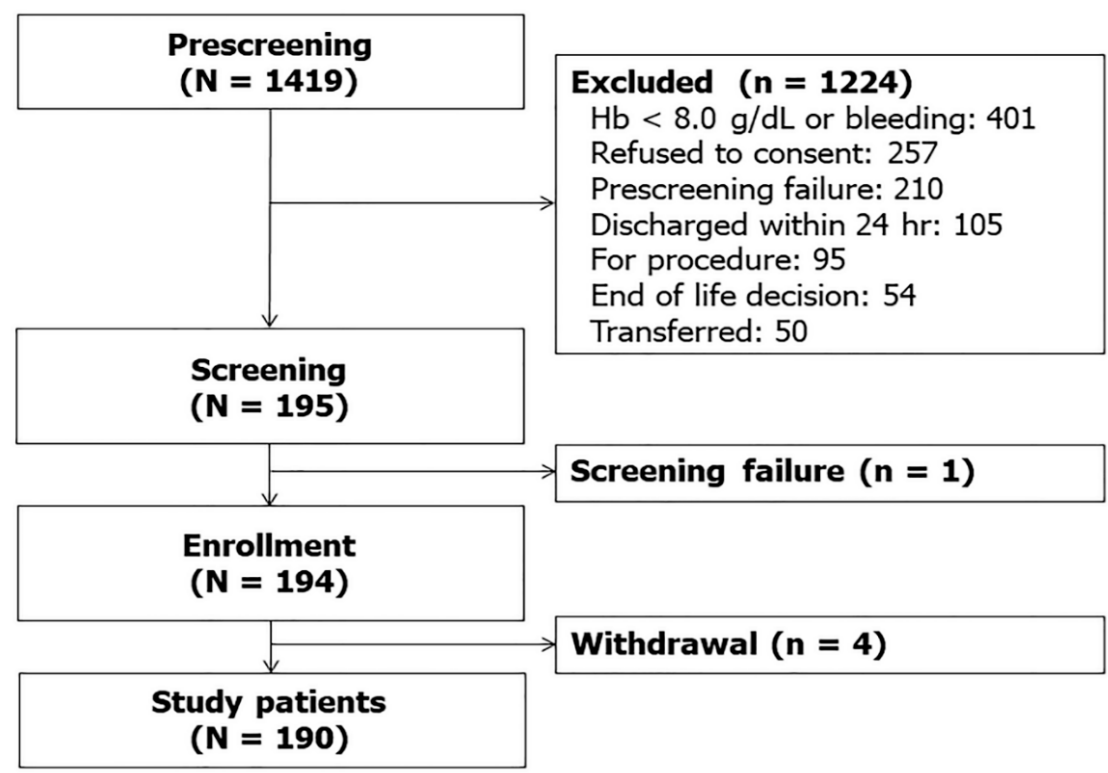

Figure 1. Study flow diagram. 
The baseline characteristics of 190 patients at the time of ICU admission are summarized in Table 1. The median age was 64 (IQR, 54-73) years, and 121 (63.7\%) patients were male. Of $62(32.6 \%)$ patients with cancer, 32 patients had hematologic malignancy. The reason for ICU admission was sepsis in $59(31.1 \%)$ patients and septic shock in $84(44.2 \%)$ patients. For 47 patients of the control group, the most common cause of ICU admission was pulmonary edema in nine patients, followed by airway stenosis in six, interstitial lung disease in five, multiple trauma in five, and acute exacerbation of chronic obstructive pulmonary disease or asthma in four patients. The median revised trauma score for five patients with multiple trauma was 13.26 (IQR, 7.19-22.15). No patients in the control group were with infection. Ninety-five (50.0\%) patients were on mechanical ventilator support, and 116 (61.1\%) patients required vasopressor support upon ICU admission. The median plasma TRAIL level was $34.48 \mathrm{pg} / \mathrm{mL}$ in the study patients. While patients on mechanical ventilatory support were more common in the control group, patients requiring vasopressor support were more prevalent in the sepsis group. Furthermore, severity scores of SAPS 3 and SOFA, as well as the C-reactive protein level were higher in the sepsis group. However, no difference in 28-day ICU mortality or in-hospital mortality was observed. The plasma TRAIL level for 143 patients with either sepsis or septic shock was $31.55 \mathrm{pg} / \mathrm{mL}$, which was lower compared to that of $52.00 \mathrm{pg} / \mathrm{mL}$ in the control group.

Table 1. Baseline characteristics of study patients $(n=190)$.

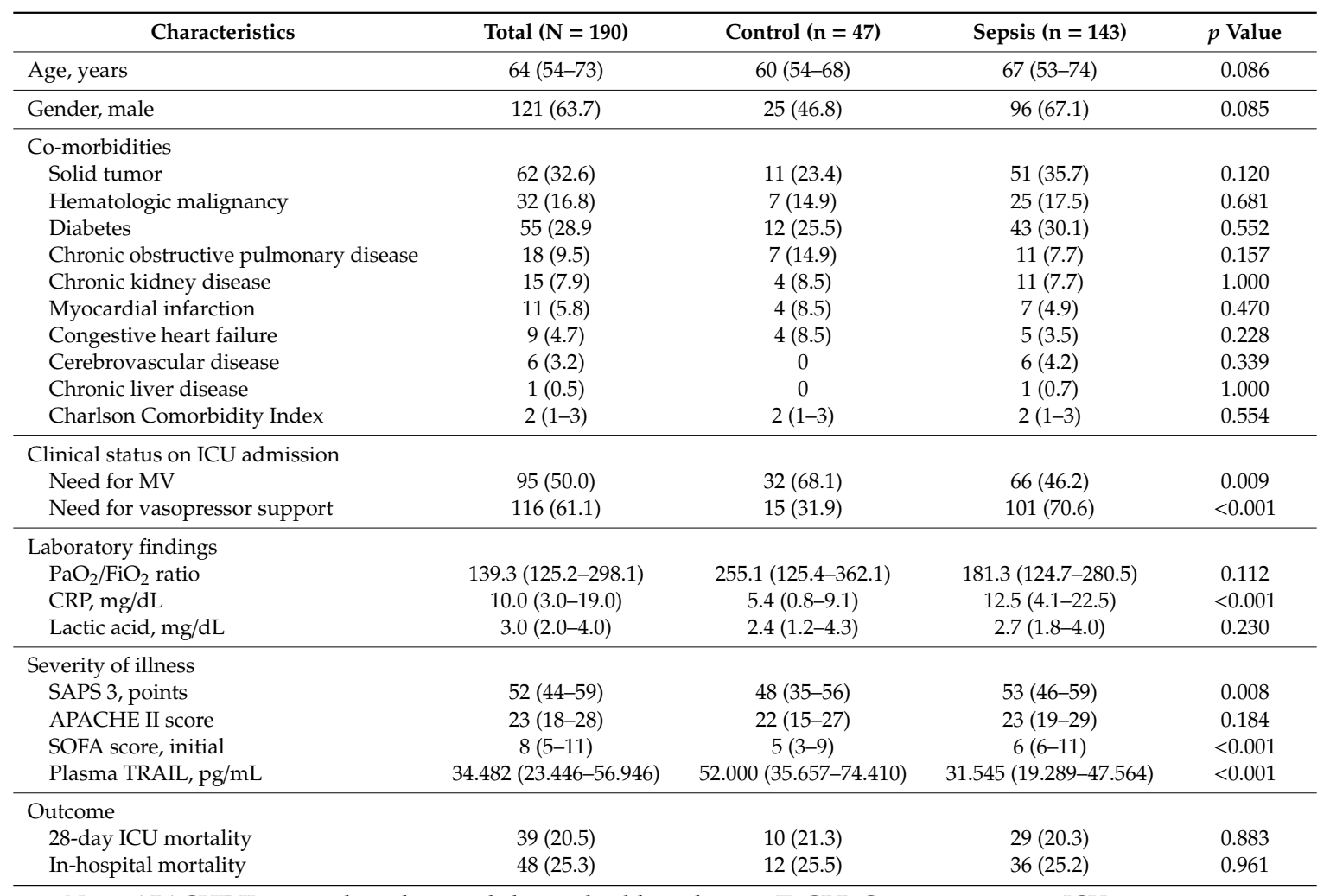

Note: APACHE II, acute physiology and chronic health evaluation II; CRP, C-reactive protein; ICU, intensive care unit; MV, mechanical ventilation; SAPS 3, simplified acute physiology score 3; SOFA, sequential organ failure assessment; TRAIL, tumor necrosis factor-related apoptosis-inducing ligand.

There was a statistically significant trend of decreased plasma TRAIL level across control, sepsis, and septic shock groups (52.00 [35.66-74.41] pg/mL vs. 35.83 [28.07-60.82] pg/mL vs. 26.08 [15.29-40.97] $\mathrm{pg} / \mathrm{mL} ; p<0.001$ ) (Figure 2). When correlation between TRAIL and RIPK3 was assessed, the plasma level of TRAIL was inversely related to the plasma level of RIPK3 in patients with sepsis and septic $\operatorname{shock}\left(\mathrm{r}=-0.172, \mathrm{r}^{2}=0.02973, p=0.039\right)$ (Figure 3). 


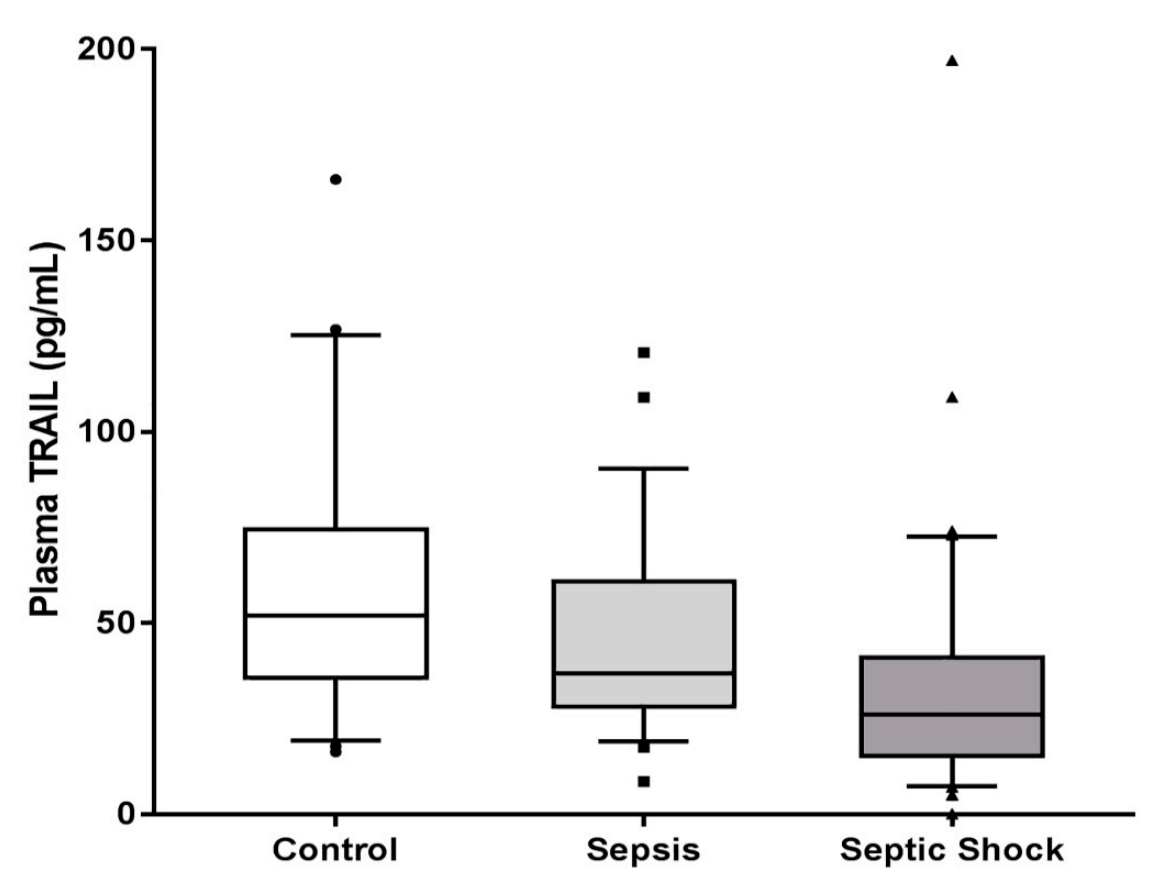

Figure 2. Plasma levels of Tumor necrosis factor TNF-related apoptosis-inducing ligand (TRAIL) in control, sepsis, and septic shock ( $p$ for trend $<0.001$ ).

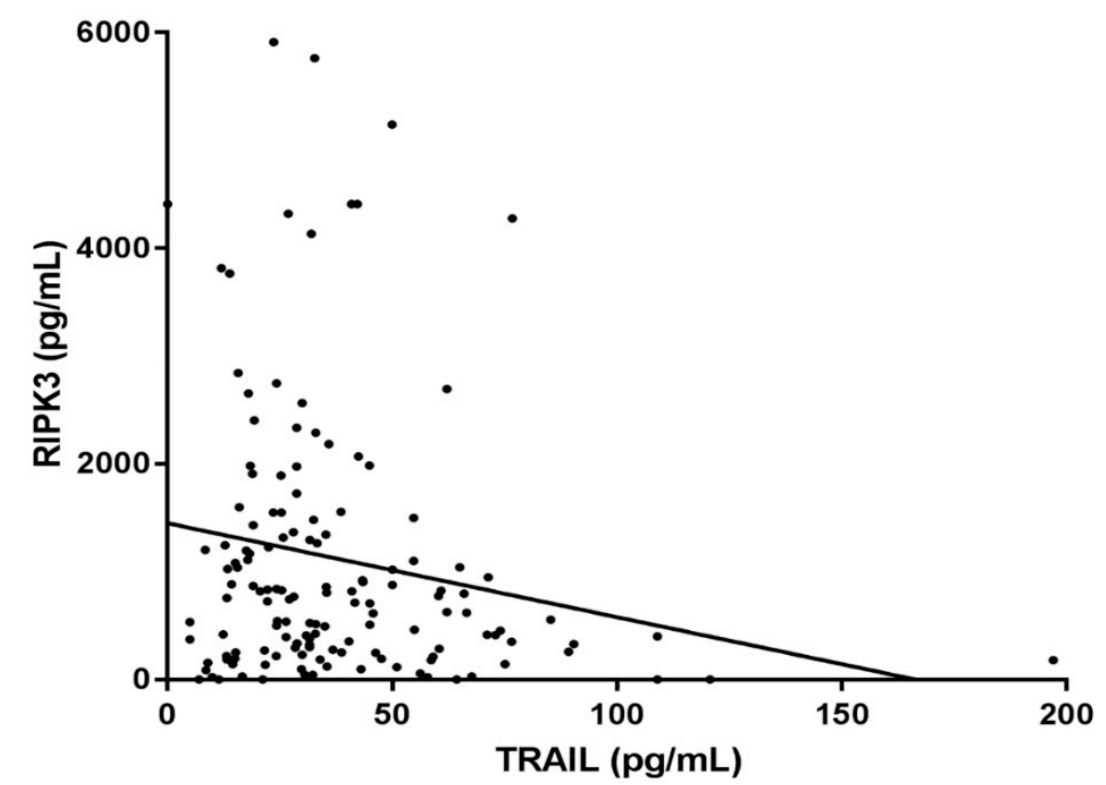

Figure 3. Correlation between plasma level of TRAIL and RIPK3 in patients with sepsis $(\mathrm{n}=143)$. Slope: -8.713 (95\% CI: -0.426--17.000), r²: 0.02973, Pearson's r: $-0.172(p=0.039)$.

To evaluate the association between characteristics of patients with sepsis and plasma TRAIL level, 143 patients who were diagnosed with either sepsis or septic shock were divided into two groups-low and high plasma TRAIL level-according to the median TRAIL level (Table 2). Septic shock and patients requiring vasopressor support were more prevalent in the group with low plasma TRAIL. Furthermore, lactic acid, the SAPS 3 score, APACHE II score, and SOFA score at initial ICU admission were significantly higher in patients with low plasma TRAIL. However, statistical differences in 28-day ICU mortality, in-hospital mortality, and 90-day mortality were not observed between the two groups. In addition, Kaplan-Meier survival estimation did not demonstrate a difference in 90-day survival between patients with high and low plasma TRAIL level $(p=0.419)$ (Figure 4). 
Table 2. Characteristics of patients with sepsis stratified according to the median level of plasma TRAIL $(\mathrm{n}=143)$.

\begin{tabular}{|c|c|c|c|}
\hline Characteristics & Low TRAIL $(n=72)$ & High TRAIL $(n=71)$ & $p$ Value \\
\hline Age, years & $67(55-73)$ & $62(51-74)$ & 0.418 \\
\hline Gender, male & $40(69.4)$ & $46(64.8)$ & 0.553 \\
\hline \multicolumn{4}{|l|}{ Co-morbidities } \\
\hline Solid tumor & $10(13.9)$ & $16(22.5)$ & 0.448 \\
\hline Hematologic malignancy & $14(19.4)$ & $11(15.5)$ & 0.534 \\
\hline Diabetes & $23(31.9)$ & $20(28.2)$ & 0.623 \\
\hline Chronic kidney disease & $11(11.6)$ & $7(7.4)$ & 0.322 \\
\hline Myocardial infarction & $8(11.1)$ & $4(5.6)$ & 0.217 \\
\hline Congestive heart failure & $3(4.2)$ & $3(4.2)$ & 1.000 \\
\hline Charlson Comorbidity Index & $2(1-3)$ & $2(1-3)$ & 0.668 \\
\hline Septic shock & $52(72.2 \%)$ & $32(45.1 \%)$ & 0.001 \\
\hline \multicolumn{4}{|l|}{ Clinical status on ICU admission } \\
\hline Need for MV & $36(50.0)$ & $30(42.3)$ & 0.353 \\
\hline Need for vasopressor support & $61(84.7)$ & $40(56.3)$ & $<0.001$ \\
\hline \multicolumn{4}{|l|}{ Laboratory findings } \\
\hline $\mathrm{PaO}_{2} / \mathrm{FiO}_{2}$ ratio & $191.7(120.2-282.6)$ & $180.6(130.5-277.5)$ & 0.436 \\
\hline $\mathrm{CRP}, \mathrm{mg} / \mathrm{dL}$ & $13.7(4.2-13.7)$ & $12.0(4.0-22.5)$ & 0.305 \\
\hline Lactic acid, mg/dL & $3.1(2.1-4.5)$ & $2.3(1.7-3.3)$ & $<0.001$ \\
\hline \multicolumn{4}{|l|}{ Severity of illness } \\
\hline SAPS 3, points & $56(50-68)$ & $50(42-56)$ & $<0.001$ \\
\hline APACHE II score & $24(19-30)$ & $22(19-27)$ & 0.007 \\
\hline SOFA score, initial & $9(7-11)$ & $7(5-11)$ & $<0.001$ \\
\hline \multicolumn{4}{|l|}{ Outcome } \\
\hline 28-day ICU mortality & $12(16.7)$ & $17(23.9)$ & 0.279 \\
\hline In-hospital mortality & $19(26.4)$ & $17(23.9)$ & 0.736 \\
\hline 90-day mortality & $23(31.9)$ & $26(36.6)$ & 0.556 \\
\hline
\end{tabular}

Note: APACHE II, acute physiology and chronic health evaluation II; CRP, C-reactive protein; ICU, intensive care unit; MV, mechanical ventilation; SAPS 3, simplified acute physiology score 3; SOFA, sequential organ failure assessment; TRAIL, tumor necrosis factor-related apoptosis-inducing ligand.

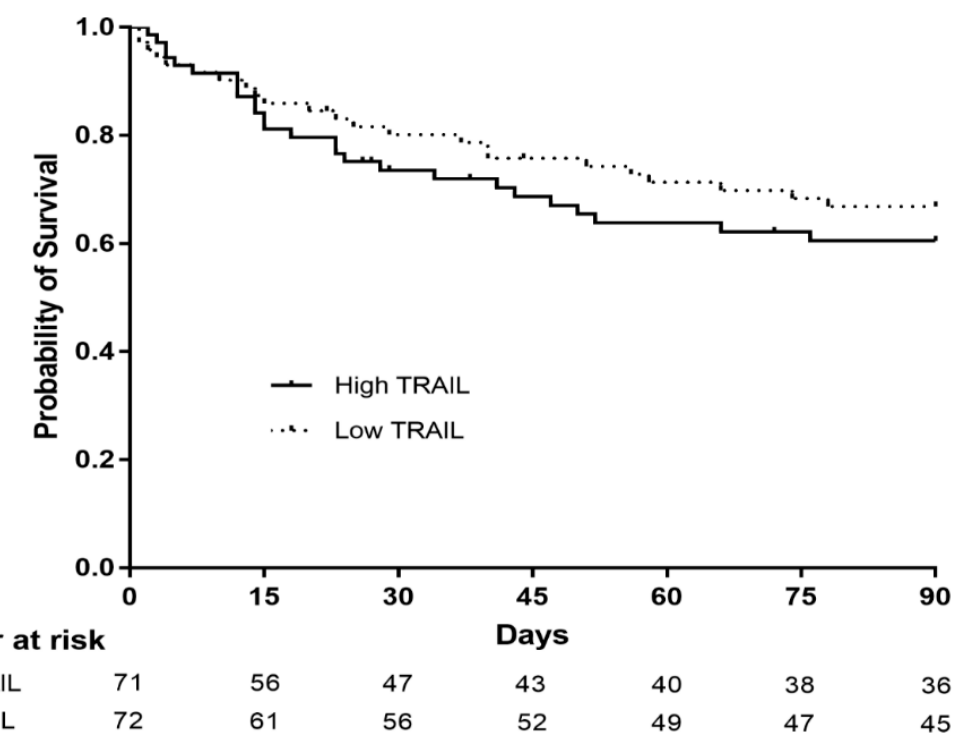

Figure 4. Kaplan-Meier survival analysis comparing patients with sepsis or septic shock with high and low plasma level of TRAIL ( $\mathrm{n}=143$ ). The 30-day and 90 -day survival estimates are $76.6 \%$ and $60.5 \%$, respectively for patients with high TRAIL (solid line), while it is $80.2 \%$ and $66.9 \%$ for patients with low TRAIL (dotted line) ( $p=0.419$, log-rank test). 
Finally, serial levels of plasma TRAIL were analyzed in 143 patients with sepsis. Blood samples at days 3 and 7 were available for TRAIL measurement in 59 and 32 patients, respectively. Plasma TRAIL levels increased over time, with median levels of $31.55 \mathrm{pg} / \mathrm{mL}(19.29-47.56)$ at day $0,40.00 \mathrm{pg} / \mathrm{mL}$ $(27.00-56.00)$ at day 3 , and $40.50 \mathrm{pg} / \mathrm{mL}(25.00-53.75)$ at day $7(p<0.001)$ (Figure 5A). Although the TRAIL level on day 3 was significantly higher compared to that of day $0(p=0.036)$, there was no difference in levels between days 3 and 7. Plasma TRAIL levels were also assessed according to 28-day ICU mortality (Figure 5B). While serial levels of TRAIL significantly increased over time in survivors $(p=0.005)$, TRAIL levels in non-survivors did not demonstrate any differences $(p=0.102)$. The TRAIL level in survivors was higher on day 3 compared to day $0(p=0.039)$; however, TRAIL levels of day 3 and day 7 did not differ. Conversely, there was no difference in levels of TRAIL between day 0 and day 3 , or between day 3 and day 7 in non-survivors. Finally, when plasma TRAIL levels on days 3 and 7 were compared between survivors and non-survivors, there was a tendency for a higher TRAIL level only on day 3 in survivors compared to non-survivors [42.00 (32.00-57.00) vs. 26.00 (17.50-53.50), $p=0.0502]$. Nonetheless, the day 7 levels demonstrated no difference between survivors and non-survivors [40.00 (25.00-52.50) vs. $53.00(23.00-75.00), p=0.801]$.

A

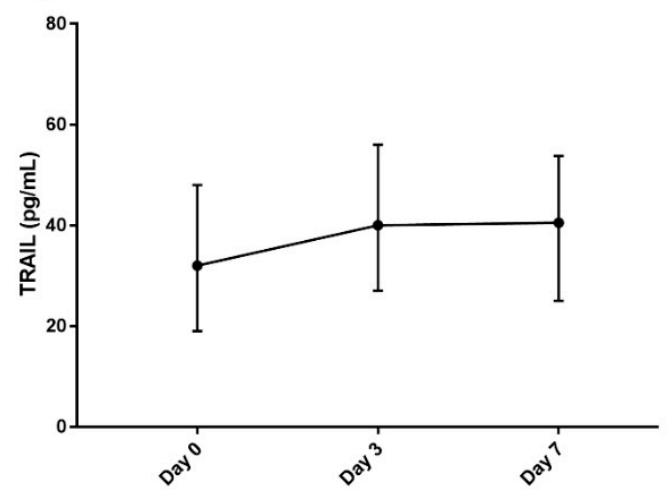

B

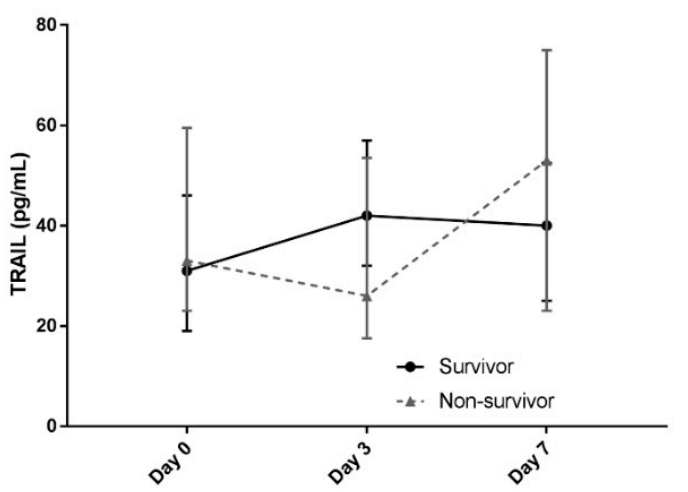

Figure 5. (A) Serial plasma levels of TRAIL on days 0,3 , and 7 of 143 patients with sepsis. (B) Comparison of serial plasma TRAIL levels in 28-day intensive care unit survivors (solid line) and non-survivors (dotted line). Upper and lower bars represent the 75th and 25th interquartile ranges, respectively.

\section{Discussion}

The aim of this prospective observational study was to investigate the association between plasma TRAIL level and sepsis severity, as well as to determine the role of TRAIL in predicting mortality. In addition, we planned to analyze the correlation between plasma levels of TRAIL and RIPK3 to identify the potential relationship of TRAIL with necroptosis. Our study of 190 consecutively enrolled critically ill patients demonstrated that the plasma TRAIL level was inversely associated with sepsis severity and plasma level of RIPK3; however, it was not predictive of mortality.

Until now, only two studies have investigated the significance of plasma TRAIL in sepsis. Tian et al. reported that the level of soluble TRAIL was lower in patients with septic shock (defined by an old definition) compared to healthy controls or patients with sepsis; soluble TRAIL was also associated with mortality [12]. Very recently, a multicenter study demonstrated an inverse correlation between the level of circulating TRAIL and both organ dysfunction and RIPK3; [13] however, the association of TRAIL with mortality was portrayed in only two of three cohorts. The inverse relationship between plasma TRAIL level and sepsis severity observed in our study concurs with the results of two previous studies, strengthening the growing evidence of the potential role of TRAIL in sepsis.

The reasons for the inverse relationship between TRAIL and sepsis severity and the role of TRAIL in sepsis pathogenesis are not fully understood. Nonetheless, there are several suggested mechanisms to explain this phenomenon. One possible explanation is based on the anti-inflammatory effect of TRAIL. TRAIL is thought to facilitate and accelerate apoptosis of leukocytes, contributing to 
resolution of inflammation [20]. Animal studies have shown that TRAIL is associated with improved survival in murine models of infection and autoimmune diseases [21-23]. However, this protective anti-inflammatory effect remains controversial. In a study by Unsinger et al. on murine cecal ligation and puncture models, TRAIL-deficient mice were better able to control bacterial infection due to TRAIL-dependent immune suppression and immune unresponsiveness [24,25]. In addition, plasma TRAIL level was measured at the early phase of sepsis in our studies, as well as previous studies. Since TRAIL-dependent apoptosis affects immune suppression and immune unresponsiveness, which plays a role in the relatively later course of sepsis [25], changes in plasma TRAIL level may not be reflective of immune status. Considering those facts, the anti-inflammatory effect of TRAIL may not fully explain its association with the pathogenesis of sepsis. Another possible mechanism is infection clearance by the TRAIL-dependent pathway. In vitro studies have shown that TRAIL contributes to infection control by mediating elimination of infected cells or restriction of viral replication in influenza or encephalomyocarditis viral infection models [26,27]. However, this observation is also conflicting. Cardoso Alves et al. demonstrated in their study that TRAIL depletion resulted in reduced NK-cell mediated antiviral $\mathrm{CD} 8^{+} \mathrm{T}$ cell killing, thereby leading to faster pathogen clearance in a mouse model of viral infection [28]. Moreover, since pathogen clearance by T cell mediated cell death is crucial in viral infection but not in bacterial infection, it may not fully explain the results of our finding. Finally, the capability of TRAIL to initiate necroptosis, a programmed cell death mechanism, may be another possible theory [10]. Although the precise function and effect of necroptosis in sepsis has yet to be elucidated, the detrimental contribution of necroptosis is thought to lie in cell death, leading to local tissue injury and excess release of pro-inflammatory DAMPs. DAMPs stimulate the innate immune response and trigger an inflammatory cascade, resulting in organ damage [15,29-31]. The relationship between sepsis and necroptosis has been observed in murine models, as well as in critically ill patients. Recent studies examining the necroptosis regulators of RIPK1, RIPK3, and MLKL in patients with sepsis reported that their levels were associated with disease severity and/or mortality [32,33]. In certain pathologic environments, such as acidic states, TRAIL triggers necroptosis via RIPK1 and RIPK3 to hinder caspase-dependent apoptosis [10,34]. Our study, as well as the study by Schenck et al. [13], illustrated a significant correlation between the plasma levels of TRAIL and RIPK3, proposing the possibility that TRAIL may have an impact on sepsis through the necroptosis pathway. Nonetheless, it should be noted that this correlation between the TRAIL and RIPK3 proteins may not provide direct evidence that TRAIL reflects the process of necroptosis of sepsis. Although we have identified in our preliminary analysis that the plasma level of TRAIL also correlates with that of mitochondrial DNA (a well-known DAMP) in sepsis (Figures S1-S3), supporting the hypothesis that TRAIL is associated with necroptosis, further research to investigate the pathogenesis of TRAIL in sepsis would be reasonable.

Contrary to previous studies, the plasma TRAIL level was not associated with either 28-day ICU mortality or in-hospital mortality in our study. Interestingly, in the study by Schenck et al. [13], the association between the plasma level of TRAIL with mortality was depicted only in two American cohorts of the United States, but not in a Korean cohort. The authors speculated that different baseline characteristics or processes of care might have contributed to this discrepancy. However, definite causes could not be determined. No relationship between TRAIL and mortality was observed in our cohort, in addition to another Korean cohort studied by Schenck et al. [13]. This raises an ethnic difference as one of the many possibilities. Since the mortality of patients with sepsis may be attributable to various factors, further studies are warranted to confirm the relationship between mortality and TRAIL level and to further investigate the reason for the current discrepancy.

One noteworthy finding of this study is that serial change in plasma TRAIL levels was available for analysis. To our knowledge, this is the first study to provide trends of the plasma TRAIL in sepsis. In particular, two discoveries require attention. First, TRAIL levels increased over time; however, the levels between days 3 and 7 did not differ. Second, when survivors and non-survivors were divided, the plasma TRAIL level on day 3 compared to day 0 was higher in survivors, but not in non-survivors. 
That there was no difference between levels on days 3 and 7 suggests that TRAIL, or the mechanism underlying the decrease in TRAIL, stabilizes or recovers within the initial days of sepsis. This suggests that TRAIL may be important in the relatively early phase of sepsis. Furthermore, because the TRAIL level on day 3 increased in survivors but not in non-survivors, perhaps it is not only the initial TRAIL level, but also the early serial changes of TRAIL that are crucial in understanding the patient's course and predicting mortality. However, since the day 3 and 7 samples were available only in some of the 143 patients with sepsis, we could not draw a definitive conclusion. As we have limited understanding of the role of TRAIL in sepsis, changes in TRAIL levels may be a consequence of sepsis or organ failure, rather than demonstration of a key process underlying sepsis. Comprehension of the pathogenesis and kinetics of TRAIL in sepsis is required to determine the significance of serial changes in TRAIL.

To fully appreciate the results of our study, several limitations should be acknowledged. First, our study was conducted at a single referral center, which may limit the generalizability of our data. Second, follow-up data on TRAIL were not available in some patients due to death, recovery, and refusal to collect additional samples. Third, based on our study protocol, enrollment of a study patient and sampling of blood were done within $24 \mathrm{~h}$ of ICU admission and $48 \mathrm{~h}$ of enrollment, respectively. Therefore, patients with high severity and early mortality may not have been included in the study. Interpretation of results requires caution, since there may be a selection bias. Fourth, the correlation between TRAIL and RIPK3 and its association with severity of sepsis does not provide sufficient evidence that TRAIL functions as an initiator of necroptosis in sepsis or necroptosis is activated in sepsis. Further studies regarding gene expression and quantification are necessary to confirm this relationship.

\section{Conclusions}

In conclusion, plasma TRAIL level was inversely associated with sepsis severity and plasma level of RIPK3. Plasma TRAIL levels increased following the course of recovery. However, they were not predictive of mortality.

Supplementary Materials: The following are available online at http://www.mdpi.com/2077-0383/9/6/1661/s1, Association of plasma level of TNF-related apoptosis inducing ligand with mitochondrial DNA in sepsis. Figure S1: Correlation between plasma level of TRAIL and mtDNA in patients with 49 patients with sepsis, Figure S2: Correlation between plasma level of RIPK3 and mtDNA in patients with 49 patients with sepsis, Figure S3: Plasma levels of mtDNA in sepsis and septic shock.

Author Contributions: H.Y. collected and analyzed the data and drafted this manuscript. J.Y.L. conducted experiments and analyzed the data. J.P. collected and analyzed the data. J.H.Y. and G.Y.S. analyzed the data and revised the manuscript. K.J. conceived and designed this study, analyzed the data, and wrote the final manuscript. All authors have read and agreed to the published version of the manuscript.

Funding: This work was supported by a Samsung Medical Center grant (SMO1190131).

Conflicts of Interest: The authors disclose that they do not have any conflict of interest.

\section{Abbreviations}

APACHE II: Acute physiology, age, chronic health evaluation II; DAMPs: Damage-associated molecular patterns; ICU: Intensive care unit; RIPK3: Receptor-interacting protein kinase-3; SAPS3: Simplified acute physiology score 3; SOFA: Sequential organ failure assessment; TRAIL, Tumor necrosis factor related apoptosis-inducing ligand.

\section{References}

1. Singer, M.; Deutschman, C.S.; Seymour, C.W.; Shankar-Hari, M.; Annane, D.; Bauer, M.; Bellomo, R.; Bernard, G.R.; Chiche, J.D.; Coopersmith, C.M.; et al. The Third International Consensus Definitions for Sepsis and Septic Shock (Sepsis-3). JAMA 2016, 315, 801-810. [CrossRef]

2. Reinhart, K.; Daniels, R.; Kissoon, N.; Machado, F.R.; Schachter, R.D.; Finfer, S. Recognizing Sepsis as a Global Health Priority-A WHO Resolution. N. Engl. J. Med. 2017, 377, 414-417. [CrossRef]

3. Rhodes, A.; Evans, L.E.; Alhazzani, W.; Levy, M.M.; Antonelli, M.; Ferrer, R.; Kumar, A.; Sevransky, J.E.; Sprung, C.L.; Nunnally, M.E.; et al. Surviving Sepsis Campaign: International Guidelines for Management of Sepsis and Septic Shock: 2016. Crit. Care Med. 2017, 45, 486-552. [CrossRef] 
4. Phua, J.; Koh, Y.; Du, B.; Tang, Y.Q.; Divatia, J.V.; Tan, C.C.; Gomersall, C.D.; Faruq, M.O.; Shrestha, B.R.; Gia Binh, N.; et al. Management of severe sepsis in patients admitted to Asian intensive care units: Prospective cohort study. BMJ 2011, 342, d3245. [CrossRef]

5. Yang, Y.; Xie, J.; Guo, F.; Longhini, F.; Gao, Z.; Huang, Y.; Qiu, H. Combination of C-reactive protein, procalcitonin and sepsis-related organ failure score for the diagnosis of sepsis in critical patients. Ann. Intensive Care 2016, 6, 51. [CrossRef] [PubMed]

6. Simon, L.; Saint-Louis, P.; Amre, D.K.; Lacroix, J.; Gauvin, F. Procalcitonin and C-reactive protein as markers of bacterial infection in critically ill children at onset of systemic inflammatory response syndrome. Pediatr Crit. Care Med. 2008, 9, 407-413. [CrossRef] [PubMed]

7. Pierrakos, C.; Vincent, J.L. Sepsis biomarkers: A review. Crit. Care 2010, 14, R15. [CrossRef] [PubMed]

8. Condotta, S.A.; Cabrera-Perez, J.; Badovinac, V.P.; Griffith, T.S. T-cell-mediated immunity and the role of TRAIL in sepsis-induced immunosuppression. Crit. Rev. Immunol. 2013, 33, 23-40. [CrossRef] [PubMed]

9. Janssen, E.M.; Droin, N.M.; Lemmens, E.E.; Pinkoski, M.J.; Bensinger, S.J.; Ehst, B.D.; Griffith, T.S.; Green, D.R.; Schoenberger, S.P. CD4+ T-cell help controls CD8+ T-cell memory via TRAIL-mediated activation-induced cell death. Nature 2005, 434, 88-93. [CrossRef]

10. Jouan-Lanhouet, S.; Arshad, M.I.; Piquet-Pellorce, C.; Martin-Chouly, C.; Le Moigne-Muller, G.; Van Herreweghe, F.; Takahashi, N.; Sergent, O.; Lagadic-Gossmann, D.; Vandenabeele, P.; et al. TRAIL induces necroptosis involving RIPK1/RIPK3-dependent PARP-1 activation. Cell Death Differ. 2012, 19, 2003-2014. [CrossRef]

11. Kaczmarek, A.; Vandenabeele, P.; Krysko, D.V. Necroptosis: The release of damage-associated molecular patterns and its physiological relevance. Immunity 2013, 38, 209-223. [CrossRef] [PubMed]

12. Tian, Y.; Tao, T.; Zhu, J.; Zou, Y.; Wang, J.; Li, J.; Bo, L.; Deng, X. Soluble tumor necrosis factor related apoptosis inducing ligand level as a predictor of severity of sepsis and the risk of mortality in septic patients. PLoS ONE 2013, 8, e82204. [CrossRef]

13. Schenck, E.J.; Ma, K.C.; Price, D.R.; Nicholson, T.; Oromendia, C.; Gentzler, E.R.; Sanchez, E.; Baron, R.M.; Fredenburgh, L.E.; Huh, J.W.; et al. Circulating cell death biomarker TRAIL is associated with increased organ dysfunction in sepsis. JCI Insight 2019, 4, e127143. [CrossRef] [PubMed]

14. Newton, K.; Dugger, D.L.; Wickliffe, K.E.; Kapoor, N.; de Almagro, M.C.; Vucic, D.; Komuves, L.; Ferrando, R.E.; French, D.M.; Webster, J.; et al. Activity of protein kinase RIPK3 determines whether cells die by necroptosis or apoptosis. Science 2014, 343, 1357-1360. [CrossRef] [PubMed]

15. Ma, K.C.; Schenck, E.J.; Siempos, I.I.; Cloonan, S.M.; Finkelsztein, E.J.; Pabon, M.A.; Oromendia, C.; Ballman, K.V.; Baron, R.M.; Fredenburgh, L.E.; et al. Circulating RIPK3 levels are associated with mortality and organ failure during critical illness. JCI Insight 2018, 3, e99692. [CrossRef]

16. Knaus, W.A.; Draper, E.A.; Wagner, D.P.; Zimmerman, J.E. APACHE II: A severity of disease classification system. Crit. Care Med. 1985, 13, 818-829. [CrossRef]

17. Moreno, R.P.; Metnitz, P.G.; Almeida, E.; Jordan, B.; Bauer, P.; Campos, R.A.; Iapichino, G.; Edbrooke, D.; Capuzzo, M.; Le Gall, J.R.; et al. SAPS 3-From evaluation of the patient to evaluation of the intensive care unit. Part 2: Development of a prognostic model for hospital mortality at ICU admission. Intensive Care Med. 2005, 31, 1345-1355. [CrossRef]

18. Vincent, J.L.; Moreno, R.; Takala, J.; Willatts, S.; De Mendonca, A.; Bruining, H.; Reinhart, C.K.; Suter, P.M.; Thijs, L.G. The SOFA (Sepsis-related Organ Failure Assessment) score to describe organ dysfunction/failure. On behalf of the Working Group on Sepsis-Related Problems of the European Society of Intensive Care Medicine. Intensive Care Med. 1996, 22, 707-710. [CrossRef]

19. Champion, H.R.; Sacco, W.J.; Copes, W.S.; Gann, D.S.; Gennarelli, T.A.; Flanagan, M.E. A revision of the Trauma Score. J. Trauma 1989, 29, 623-629. [CrossRef]

20. McGrath, E.E.; Marriott, H.M.; Lawrie, A.; Francis, S.E.; Sabroe, I.; Renshaw, S.A.; Dockrell, D.H.; Whyte, M.K. TNF-related apoptosis-inducing ligand (TRAIL) regulates inflammatory neutrophil apoptosis and enhances resolution of inflammation. J. Leukoc. Biol. 2011, 90, 855-865. [CrossRef]

21. Cretney, E.; McQualter, J.L.; Kayagaki, N.; Yagita, H.; Bernard, C.C.; Grewal, I.S.; Ashkenazi, A.; Smyth, M.J. TNF-related apoptosis-inducing ligand (TRAIL)/Apo2L suppresses experimental autoimmune encephalomyelitis in mice. Immunol. Cell Biol. 2005, 83, 511-519. [CrossRef] 
22. Hoffmann, O.; Priller, J.; Prozorovski, T.; Schulze-Topphoff, U.; Baeva, N.; Lunemann, J.D.; Aktas, O.; Mahrhofer, C.; Stricker, S.; Zipp, F.; et al. TRAIL limits excessive host immune responses in bacterial meningitis. J. Clin. Investig. 2007, 117, 2004-2013. [CrossRef]

23. Lamhamedi-Cherradi, S.E.; Zheng, S.J.; Maguschak, K.A.; Peschon, J.; Chen, Y.H. Defective thymocyte apoptosis and accelerated autoimmune diseases in TRAIL-/- mice. Nat. Immunol. 2003, 4, 255-260. [CrossRef]

24. Unsinger, J.; Kazama, H.; McDonough, J.S.; Griffith, T.S.; Hotchkiss, R.S.; Ferguson, T.A. Sepsis-induced apoptosis leads to active suppression of delayed-type hypersensitivity by CD8+ regulatory $\mathrm{T}$ cells through a TRAIL-dependent mechanism. J. Immunol. 2010, 184, 6766-6772. [CrossRef]

25. Gurung, P.; Rai, D.; Condotta, S.A.; Babcock, J.C.; Badovinac, V.P.; Griffith, T.S. Immune unresponsiveness to secondary heterologous bacterial infection after sepsis induction is TRAIL dependent. J. Immunol. 2011, 187, 2148-2154. [CrossRef]

26. Brincks, E.L.; Katewa, A.; Kucaba, T.A.; Griffith, T.S.; Legge, K.L. CD8 T cells utilize TRAIL to control influenza virus infection. J. Immunol. 2008, 181, 4918-4925. [CrossRef]

27. Sato, K.; Hida, S.; Takayanagi, H.; Yokochi, T.; Kayagaki, N.; Takeda, K.; Yagita, H.; Okumura, K.; Tanaka, N.; Taniguchi, T.; et al. Antiviral response by natural killer cells through TRAIL gene induction by IFN-alpha/beta. Eur. J. Immunol. 2001, 31, 3138-3146. [CrossRef]

28. Cardoso Alves, L.; Berger, M.D.; Koutsandreas, T.; Kirschke, N.; Lauer, C.; Sporri, R.; Chatziioannou, A.; Corazza, N.; Krebs, P. Non-apoptotic TRAIL function modulates NK cell activity during viral infection. EMBO Rep. 2020, 21, e48789. [CrossRef]

29. Yatim, N.; Cullen, S.; Albert, M.L. Dying cells actively regulate adaptive immune responses. Nat. Rev. Immunol. 2017, 17, 262-275. [CrossRef]

30. Moreno-Gonzalez, G.; Vandenabeele, P.; Krysko, D.V. Necroptosis: A Novel Cell Death Modality and Its Potential Relevance for Critical Care Medicine. Am. J. Respir. Crit. Care Med. 2016, 194, 415-428. [CrossRef]

31. Kirkpatrick, A.W.; Coccolini, F.; Ansaloni, L.; Roberts, D.J.; Tolonen, M.; McKee, J.L.; Leppaniemi, A.; Faris, P.; Doig, C.J.; Catena, F.; et al. Closed Or Open after Source Control Laparotomy for Severe Complicated Intra-Abdominal Sepsis (the COOL trial): Study protocol for a randomized controlled trial. World J. Emerg. Surg. 2018, 13, 26. [CrossRef] [PubMed]

32. Wang, B.; Li, J.; Gao, H.M.; Xing, Y.H.; Lin, Z.; Li, H.J.; Wang, Y.Q. Necroptosis regulated proteins expression is an early prognostic biomarker in patient with sepsis: A prospective observational study. Oncotarget 2017, 8, 84066-84073. [CrossRef] [PubMed]

33. Vucur, M.; Roderburg, C.; Kaiser, L.; Schneider, A.T.; Roy, S.; Loosen, S.H.; Luedde, M.; Trautwein, C.; Koch, A.; Tacke, F.; et al. Elevated Serum Levels of Mixed Lineage Kinase Domain-Like Protein Predict Survival of Patients during Intensive Care Unit Treatment. Dis. Markers 2018, 2018, 1983421. [CrossRef] [PubMed]

34. Gonzalvez, F.; Ashkenazi, A. New insights into apoptosis signaling by Apo2L/TRAIL. Oncogene 2010, 29, 4752-4765. [CrossRef]

(C) 2020 by the authors. Licensee MDPI, Basel, Switzerland. This article is an open access article distributed under the terms and conditions of the Creative Commons Attribution (CC BY) license (http://creativecommons.org/licenses/by/4.0/). 